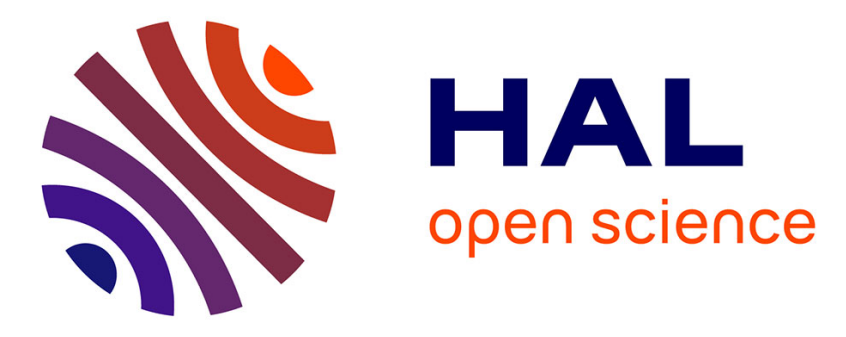

\title{
Amplitude Control of an Ultrasonic Vibration for a Tactile Stimulator
}

Wael Ben Messaoud, Frédéric Giraud, Betty Lemaire-Semail, Michel Amberg, Marie-Ange Bueno

\section{> To cite this version:}

Wael Ben Messaoud, Frédéric Giraud, Betty Lemaire-Semail, Michel Amberg, Marie-Ange Bueno. Amplitude Control of an Ultrasonic Vibration for a Tactile Stimulator. IEEE/ASME Transactions on Mechatronics, 2016, 21 (3), pp.1692 - 1701. 10.1109/TMECH.2016.2535300 . hal-01396831

\section{HAL Id: hal-01396831 \\ https://inria.hal.science/hal-01396831}

Submitted on 26 Sep 2018

HAL is a multi-disciplinary open access archive for the deposit and dissemination of scientific research documents, whether they are published or not. The documents may come from teaching and research institutions in France or abroad, or from public or private research centers.
L'archive ouverte pluridisciplinaire HAL, est destinée au dépôt et à la diffusion de documents scientifiques de niveau recherche, publiés ou non, émanant des établissements d'enseignement et de recherche français ou étrangers, des laboratoires publics ou privés. 


\title{
Amplitude control of an ultrasonic vibration for a tactile stimulator
}

\author{
Wael Ben Messaoud, Frédéric Giraud, Member, IEEE, Betty Lemaire-Semail, Member, IEEE, Michel \\ Amberg, and Marie-Ange Bueno
}

\begin{abstract}
This paper describes the control in a (d-q) frame of the vibration amplitude of a tactile stimulator based on ultrasonic vibrations. The objective is to combine two control strategies in order to maintain a constant level of vibration amplitude and to excite at the resonance frequency of the system. A new modelling approach is presented to identify the transfer function between inputs and outputs. Then, thanks to an experimental setup, the validation of the new model is performed. Finally, the result of the closed loop control over wide range of disturbing factors is presented.
\end{abstract}

Index Terms-Vibrations, Modelling, Frequency control, Piezoelectricity, Tactile display.

\section{INTRODUCTION}

$T_{1}$ he sense of touch plays an important role during the manipulation and the interaction with an object. However, the last decade has witnessed the emergence of new electronic devices with a poor tactile feedback. For example, the tactile experience when touching today's smartphones is not very different from one device to another since most of them use a flat and hard touch screen to enter commands. Actuators are then used to create a programmable and synthetic haptic feedback; but due to their technology, they can provide low frequency vibrations only, leading to limited capabilities to encode information. However, there are other technologies which can render a more sophisticated tactile feedback by modifying the user's perception of a touched surface. In particular, tactile stimulators based on friction modulation adjust the apparent friction between the user's fingertip and the touched surface to produce a wide range of effects. Besides, the issue of high fidelity tactile feedback systems does not concern only the smartphone's market but also other fields of application such as the detection and rehabilitation of tactile disorders, the virtual prototyping, the e-commerce, etc.

W.B.M. Author is with L2EP-IRCICA Laboratory, University of Lille1, Lille, France, 59650 and LPMT Laboratory, Ecole Nationale Supérieure d'Ingénieurs Sud Alsace, University of Haute Alsace, 68093 Mulhouse, France. E-mail: wael.ben-messaoud@ed.univ-lille1.fr

F.G. Author is with L2EP-IRCICA Laboratory, University of

Lille1, Lille, France, 59650. E-mail: frederic.giraud@ polytech-lille.fr

B.L.S. Author is with L2EP-IRCICA Laboratory, University of Lille1, Lille, France, 59650. E-mail: betty.semail@polytech-lille.fr

M.A. Author is with L2EP-IRCICA Laboratory, Lille, University of Lille1, France, 59650. E-mail: michel.Amberg@univ-lille1.fr

M.A.B. is with LPMT Laboratory, Ecole Nationale Supérieure d'Ingénieurs Sud Alsace, University of Haute Alsace, 11 rue Alfred Werner, 68093 Mulhouse, France. E-mail: marie-ange.bueno@uha.fr
Two main techniques are used to control the friction; electrovibration increases the friction by creating an attractive electrostatic force to a user's finger when it is moving over a plate [1][2]. On the other hand, ultrasonic vibrations reduce the friction coefficient between the user's fingertip and he vibrating surface by creating an active lubrication of the surface. This principle has been presented at the first time in 1995 by Watanabe et al. [3] who proposed the "squeezed film air theory" to explain the friction reduction. The design of the vibrating touched surface is not straightforward, and should fulfil some requirements. Indeed, it should be large enough to allow a free movement of the finger, and needs to optimize a bending mode of the plate [4]. Moreover, the vibration amplitude should be superior to $1 \mu \mathrm{m}$ at a frequency above 25 $\mathrm{kHz}$ [5]. However, the vibration of the plate can be sensitive to external factors, such as the temperature which changes the resonant frequency of the chosen bending mode or the user's fingertip pressure which damps the vibration. A closed loop control is then needed to guarantee a constant vibration amplitude. Some previous works deal with closed loop control for piezoelectric actuator to drive high-precision stages [6]. In [7], the control of a haptic knob is presented to eliminate the effect of the finger pressure. The voltage across the piezoelectric material is adjusted in order to obtain the required vibration amplitude. In other control schemes [8][9], the piezoelectric ceramics are supplied with a constant voltage amplitude while the frequency is adjusted in order to reach the required vibration amplitude. With these methods, operation at resonance is not guaranteed, which may decrease the global efficiency of the system. Indeed, for a resonant ultrasonic device operating at fixed vibration amplitude, the required amount of voltage increases as the frequency shifts away of the resonant frequency. In our recent work [10], a first approach was proposed to cope with the two disturbing factors, the finger pressure which is measured by the force sensors in the stimulator and the room temperature variation measured by a thermometer. This first study relied on an empirical modelling of the device and the frequency control was based on a "bang-bang" strategy [11]. This approach gives very satisfactory results especially when the initial excitation frequency was close to the resonance. However, with unsuited initial condition, the bang-bang control could lead to bad performances in terms of response time.

This paper uses a more general approach to present a control of the vibration amplitude of the plate with a 
simultaneous tracking of the resonant frequency of the bending mode. It is based on the modelling and the control in a (d-q) frame of a tactile plate in order to overcome external factors such as temperature variation and finger pressure variation which weaken the vibration amplitude. This representation is used to transform reference frame into a new frame that rotates synchronously with the specified vector. The control of variables becomes easier achieved by choosing well the reference vector of the frame. The $d-q$ vector representation has been used in the case of travelling wave for a motor [12], for a standing wave piezoelectric actuator in [13], and in the case of a finite beam to control a travelling wave [14]. It has been widely used to represent and to control AC electric machines [15]; and also in the grid-connected power inverter domain to track the phase and the frequency of the grid voltage, and to control the exchange of power between the electric system and the direct current (DC) bus [16]. The tracking of the resonant frequency was used in the detection of contact in robotics application [17].

In the first part of this paper, the tactile feedback system will be presented and the influence of some external parameters on the vibration quality will be highlighted experimentally. Then, the modelling in $\mathrm{d}-\mathrm{q}$ frame will be developed in part III. Part IV will focus on the experimental identification of the model parameters. Finally, in the last part, the simultaneous control of the vibration and tracking of the resonant frequency will be presented.

\section{SYSTEM PRESENTATION}

\section{A. Design of the tactile stimulator}

Ultrasonic vibrations are used to produce friction modulation by generating a squeezed film air bearing. This vibration is non-perceptible by the cutaneous mechanoreceptors whose bandwidth is below $1 \mathrm{kHz}$ [18] but the resulting sliding effect on the plate is easily perceivable. In order to create these vibrations, piezoelectric ceramics are glued under the aluminium plate; the material of the ceramics is PZT and their dimension is $14 * 6 * 0.5 \mathrm{~mm}^{3}$. They are used as actuators by means of the inverse piezoelectric effect. For the plate studied in this paper, the size is $198 * 138 * 1.2 \mathrm{~mm}^{3}$, and 20 actuators were glued under the surface (18 are used as actuators and 2 as sensors). Actuators are arranged into four columns, two in each border. Each column is composed by five ceramics distributed over the whole width of the plate. When they are excited at the mechanical resonant frequency of the chosen bending mode, a standing wave is generated as depicted in Fig. 1. The measurement of the instantaneous vibration amplitude is carried out using two piezoelectric ceramics as sensors. They have to be positioned at the antinode of vibration and they convert the deformation of the plate into a voltage proportional to the vibration amplitude. The sensors are calibrated using a vibrometer in order to find the linear relation between the sensor response and the instantaneous vibration amplitude. The system is controlled by a DSP (Digital Signal Processor) type STM32F4 which generates the numerical control signal. This signal is then amplified by a DC-AC converter in order to produce the supply voltage to the actuators. The response of the tactile stimulator - i.e. the vibration amplitude - is measured thought the sensors of the plate. Fig. 2 describes the system.

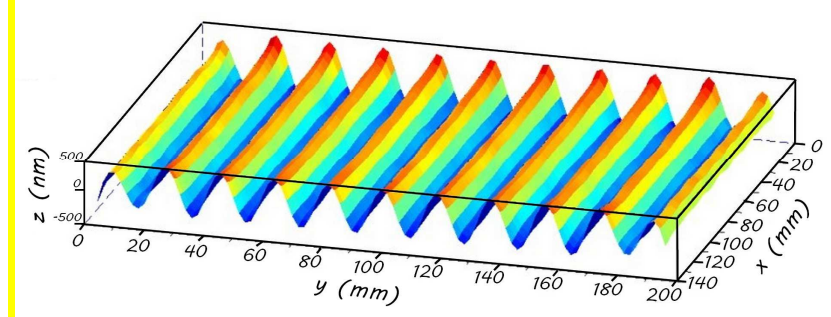

Fig. 1. The chosen bending vibration mode when the system is excited at the resonance

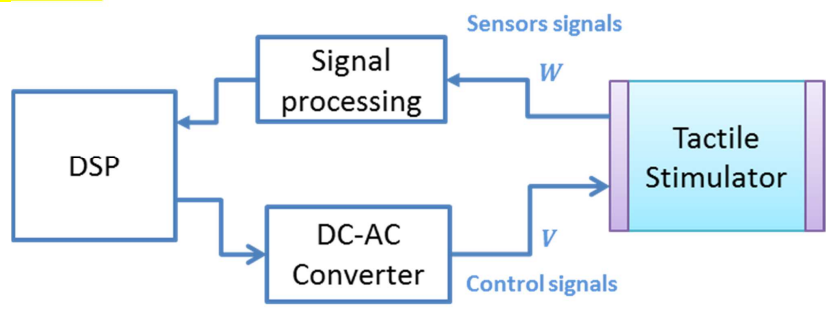

Fig. 2. Operation principle of the tactile stimulator

\section{B. Influence of external parameters}

First studies on friction reduction based tactile devices were carried out in open loop, the wave amplitude was not controlled [19][20][21]. However, in recent publications [22][10], it was highlighted that some external factors such as the finger pressure and the temperature variation can disturb those devices while modifying the vibration amplitude and the resonant frequency. The measurements of Fig. 3 show that the vibration can be damped by $50 \%$ with an applied normal force of around 1.5 N. Moreover, this variation are quite fast, and measurements in [10] have shown that this normal force could increase by $1 \mathrm{~N}$ in 0.1 second.

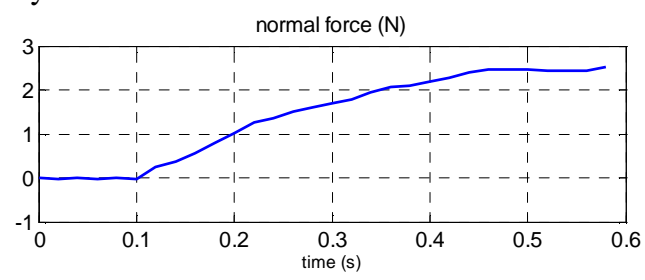

percentage of amplitude reduction (\%)

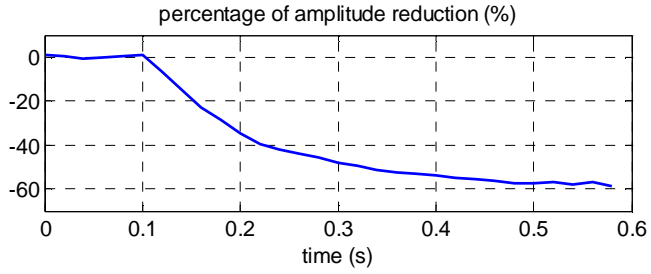

Fig. 3. Percentage of the amplitude reduction in function of the normal force

Another factor modifies the resonant frequency of the plate: the variation of the external temperature. By increasing the temperature, the mechanical structure becomes softer which decreases the stiffness parameter c leading to decreasing the resonance frequency [23]. Indeed, Fig. 4 shows the vibration 
amplitude as a function of the frequency at two different temperatures $\left(24^{\circ} \mathrm{C}\right.$ and $\left.33^{\circ} \mathrm{C}\right)$. It shows that the resonant frequency is decreased from $32.3 \mathrm{kHz}$ to $32.2 \mathrm{kHz}$ when the temperature increases. The variation of $9^{\circ} \mathrm{C}$ of temperature is supposed as the extreme possible variation in a day. This curve was plotted after putting the plate in an oven and varying the temperature from $24^{\circ} \mathrm{C}$ to $33^{\circ} \mathrm{C}$ by a step of $1^{\circ} \mathrm{C}$. In this range of temperature, a linear relation was found between the resonant frequency and the temperature.

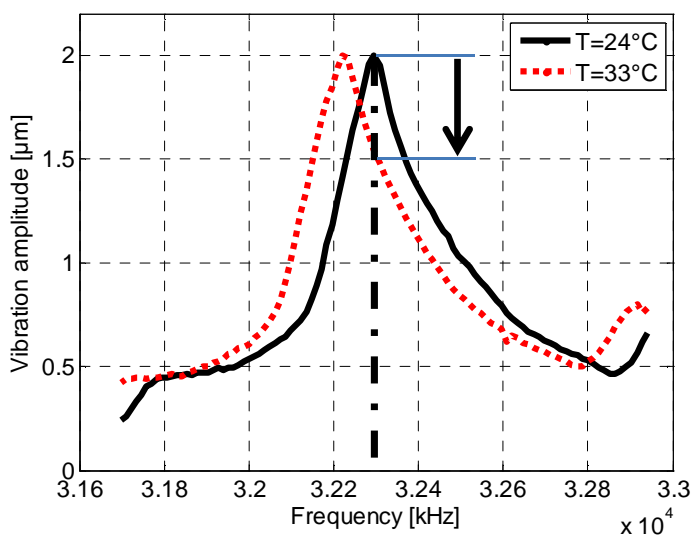

Fig. 4. Frequency response around one mode according to two temperatures: $24^{\circ} \mathrm{C}$ (continuous, black line) and $33^{\circ} \mathrm{C}$ (dotted, red line)

Hence, for a constant supply frequency adjusted to the resonant frequency at the lower temperature, the vibration amplitude decreases when temperature increases. Moreover, despite the small frequency shifts $(0.3 \%$ for the example above), the effect is not negligible, and the amplitude of vibration is reduced by $20 \%$ from its initial value. At the end, an experimental study shows that the vibration amplitude reduction may reach up to $8 \%$ for only a $4^{\circ} \mathrm{C}$ variation.

The aforementioned sensitivity to external factors leads us to propose a closed loop control of the vibration in addition to the tracking of the resonant frequency in order to obtain the robustness of the system at each operating condition. The modelling of the system in order to obtain the control law by its inversion is described in this paper.

\section{MODELLING OF THE STIMULATOR'S VIBRATION IN D-Q FRAME}

\section{A. General equations in $d$ - $q$ frame}

Around resonant frequency of the chosen bending mode, and under purely sinusoidal excitation, the evolution of the vibration amplitude $W(t)$ is given by the second type equation in the complex form [13]:

$$
m \underline{\ddot{W}}+d_{s} \underline{\dot{W}}+c \underline{W}=N \underline{V}-F
$$

with $m$ the modal mass, $d_{s}$ the modal damping, $c$ the modal stiffness, $N$ is the electromechanical conversion factor, $F$ represents the effect of the external force applied to the system, $\underline{V}$ is the input supply voltage and $\underline{W}$ is the vibration amplitude. The latter and the voltage can be represented with their complex form and we name $W_{d}$ and $W_{q}\left(V_{d}\right.$ and $\left.V_{q}\right)$ the real and imaginary parts of the vibration (and of the voltage respectively) as follows:

$$
\begin{aligned}
\underline{W} & =\left(W_{d}+j W_{q}\right) e^{j \omega t} \\
\underline{V} & =\left(V_{d}+j V_{q}\right) e^{j \omega t}
\end{aligned}
$$

Replacing $\underline{W}, \underline{\dot{W}}$ and $\underline{W}$ by their expressions in (1), assuming that the variation of $\omega$ is minor $(\dot{\omega}=0)$ and considering $F=0$ yields (4) and (5). The modelling and identification process are performed without load and $F$ is assumed as disturbance of the closed loop configuration.

$$
\begin{gathered}
m \ddot{W}_{d}+d_{s} \dot{W}_{d}+\left(c-m \omega^{2}\right) W_{d}-\omega\left(2 m \dot{W}_{q}+d_{s} W_{q}\right) \\
=N V_{d} \\
m \ddot{W}_{q}+d_{s} \dot{W}_{q}+\left(c-m \omega^{2}\right) W_{q}+\omega\left(2 m \dot{W}_{d}+d_{s} W_{d}\right) \\
=N V_{q}
\end{gathered}
$$

These two equations describe the vibratory behaviour as a function of the voltage and the excitation frequency. In order to simplify the model and because we have a degree of freedom for the choice of the d-q frame, we set $V_{d}=0$. Hence, we decide to align the rotating reference frame on the voltage vector. This can be represented by the vector representation of figure 5, where the vectors $\underline{V}$ and $\underline{W}$ are represented. It may be noted that this approach differs from the one described in [13] because axis $d$ was attached to $\underline{W}$ using a phase locked loop, leading to $W_{q}=0$, and the voltage components $V_{d}$ and $V_{q}$ were constrained by the condition of a constant voltage amplitude $\left(\sqrt{V_{d}{ }^{2}+V_{q}^{2}}=\right.$ cste $)$. This approach did not ensure operation at the resonant frequency, whereas our goal here is the control of the vibration amplitude and a real time tracking of the resonant frequency.

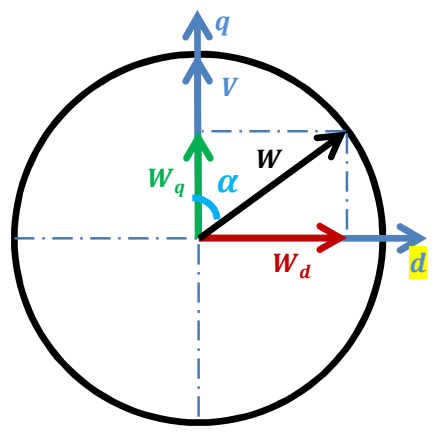

Fig. 5. Representation of the voltage and the vibration amplitude in d-q axis with $V_{d}$

\section{B. Equations at resonance}

The synchronisation of the vector $\underline{V}$ on the axis q makes $V_{d}=$ 0 . At resonance, we have $\omega_{0}^{2}=\bar{\omega}^{2}=c / m$. For steady state conditions, the derivative terms of $W_{q}$ and $W_{d}$ are zero. Then, equations (4) and (5) give the equations (6) and (7): 


$$
\begin{aligned}
& m \ddot{W}_{a}+d_{s} \dot{W}_{a}+\left(c-m \omega^{2}\right) W_{d}-\omega\left(2 m \dot{W}_{q}+d_{s} W_{q}\right) \\
& =0 \\
& m \ddot{W}_{q}+d_{s} \dot{W}_{q}+\left(c-m \omega^{z}\right) W_{q}+\omega\left(2 m \dot{W}_{a}+d_{s} W_{d}\right) \\
& =N V_{q}
\end{aligned}
$$

From the equation (6), we can conclude that the imaginary part of the vibration amplitude $W_{q}=0$ and, (7) gives a linear relation between $V_{q}$ and $W_{d}$ illustrated in the following equation (8):

$$
N V_{q}=d_{s} \omega W_{d}
$$

With this representation on $\mathrm{d}-\mathrm{q}$ frame, we are able to determine easily whether we are exciting the system at resonance or not by verifying if $W_{q}$ is null or not. So, to track the resonant frequency, two parameters are adjustable: the voltage amplitude $V$ and $\omega$ the angular frequency $(\omega=2 \pi f)$. Each of them has an influence on the real and imaginary part of vibration respectively $W_{d}$ and $W_{q}$, as the voltage is synchronized on the $\mathrm{q}$ axis. The diameter of the circle plotted on Fig. 5 is a function of the voltage amplitude $V$, if the voltage increases the diameter increases also. The variation of voltage frequency affects the angle between $\underline{W}$ and $\underline{V}$ which is $\pi / 2$ at resonance. If this angle is less than $\pi / 2$, the excitation frequency must be increased and if this angle is higher than $\pi / 2$ the excitation frequency must be decreased in order to minimize the ratio $V / W$.

\section{Transfer functions for the control}

Since $\underline{V}$ is attached to the axis q, we write $V_{d}=0$ and $V_{q}=$ $V$. This condition gives rise to the equations (9) and (10):

$$
\begin{gathered}
m \ddot{W}_{d}+d_{s} \dot{W}_{d}+\left(c-m \omega^{2}\right) W_{d}-\omega\left(2 m \dot{W}_{q}+d_{s} W_{q}\right) \\
=0 \\
m \ddot{W}_{q}+d_{s} \dot{W}_{q}+\left(c-m \omega^{2}\right) W_{q}+\omega\left(2 m \dot{W}_{d}+d_{s} W_{d}\right) \\
=N V_{q}
\end{gathered}
$$

From these equations, two working conditions can be studied: in the vicinity of the resonance, and for variation of the supply frequency.

\section{1) Modelling of the vibration amplitude in the vicinity of the resonance}

In the vicinity of resonance $W_{q}=0$, and the equation (10) can be written as follows:

$$
\omega_{0}\left(2 m \dot{W}_{d}+d_{s} W_{d}\right)=N V
$$

The resonant angular frequency $\omega_{0}$ depends on $\mathrm{c}$ and $\mathrm{m}$ :

$$
\omega_{0}=\sqrt{\frac{c}{m}}
$$

Applying Laplace transformation, we find a first order transfer function between $W_{d}$ and $V$.

$$
\frac{W_{d}(p)}{V(p)}=\frac{K_{1}}{1+\tau_{1} p}
$$

with $K_{1}=N / d_{s} \omega_{0}$ and $\tau_{1}=2 m / d_{s}$.

Hence, in the rotating reference frame, the evolution of the vibration amplitude follows a first order equation whose time constant equals $\tau_{1}$ and static gain is $K_{1}$. A PI controller can be used to control $W_{d}$ to the reference, as described in [7].

\section{2) System behaviour to frequency change}

In this section, we assume that $W_{d}$ is perfectly controlled and constant. Hence, the derivatives terms of $W_{d}$ which are $\dot{W}_{d}$ and $\ddot{W}_{d}$ will be null and equation (9) becomes:

$$
\left(c-m \omega^{2}\right) W_{d}-\omega\left(2 m \dot{W}_{q}+d_{s} W_{q}\right)=0
$$

In this equation, $\omega$ and and $W_{q}$ are variables, leading to a differential equation with non-constant parameters. For the purpose of simplification, we propose a linearization of the equation. Indeed, considering that $\omega=\omega_{0}+\Delta \omega$, We can write:

$$
c-m \omega^{2}=c\left(1-\left(1+\frac{\Delta \omega}{\omega_{0}}\right)^{2}\right)
$$

The first order Taylor approximation can be employed because the variation between the excitation frequency $\omega$ and the resonant frequency $\omega_{0}$ is very small, and $\Delta \omega \approx 0$; thus, we can write:

$$
\left(1+\frac{\Delta \omega}{\omega_{0}}\right)^{2} \approx 1+2 \frac{\Delta \omega}{\omega_{0}}
$$

By replacing (15) and (16) in (14), we have:

$$
c-m \omega^{2} \approx-2 m \omega_{0} \Delta \omega
$$

Same considerations lead us to approximate $\omega$ to $\omega_{0}$, and with (16), and (14), we obtain the equation (18):

$$
-2 m \Delta \omega W_{d}=2 m \dot{W}_{q}+d_{s} W_{q}
$$

Leading to:

$$
\frac{\left(\frac{W_{q}(p)}{W_{d}}\right)}{\Delta \omega(p)}=\frac{K_{2}}{1+\tau_{2} p}
$$

with $K_{2}=-2 m / d s$ and $\tau_{2}=2 m / d s$.

Two important remarks can be elicited from these transfer functions:

- $\tau_{1}=\tau_{2}$ : the two transfer functions (13) and (19) have the same time constant.

- This transfer function shows that changes in the supply frequency of the tactile stimulator results in $q$ change of the ratio $W_{q} / W_{d}$, according to a first order equation with still $\tau_{1}$ for time constant. Hence, to obtain the tracking of the 
resonant frequency, a PI controller is used to change the frequency in order to obtain $W_{q} / W_{d}=0$, because at resonance, $W_{q}=0$.

In the next section, the method of parameter's identification and the control of the output of these models will be investigated.

\section{EXPERIMENTAL IDENTIFICATION}

In this section, the two transfer functions previously detailed are identified by applying a step variation of the input and measuring the output in time domain. The experimental set-up necessary for the identification is described.

\section{A. Experimental setup description}

The tactile stimulator presented in II)A) was used in the identification process. It is connected to a PC through the USB port in read and write mode. The read mode allows us to display in real time different parameters with $1 \mathrm{kHz}$ sampling time, like the instantaneous vibration amplitude, the voltage amplitude and the frequency excitation. The write mode is used to send the amplitude references to the system. The DSP is powered by another USB port, See Fig. 6 .

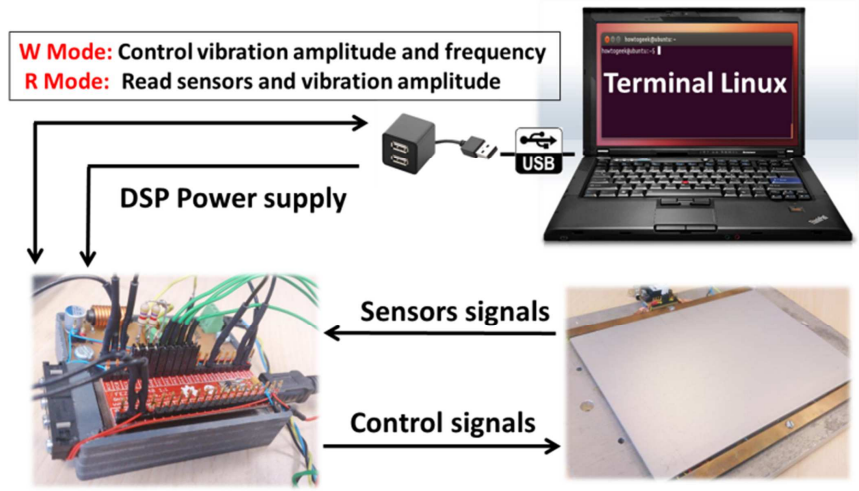

Fig. 6. Experimental setup for the identification process

\section{B. Identification approach}

1) Identification of the transfer function between $W_{d}$ and $V$

The first point is to identify the static gain $K_{1}$ of the transfer function (13). For that aim, successive values of the voltage from $50 \mathrm{~V}$ to $400 \mathrm{~V}$ peak to peak are applied to the piezoceramics at the resonant frequency, and the vibration amplitude is measured. The choice of this range of voltage is explained by the desired range of the vibration amplitude $[0.5 \ldots 2.5 \mu \mathrm{m}]$. This relation between the supply voltage and the vibration amplitude is linear in the useful range for this application. Fig. 7 shows the slope of curve which is $W_{d} / V=K_{1}$.

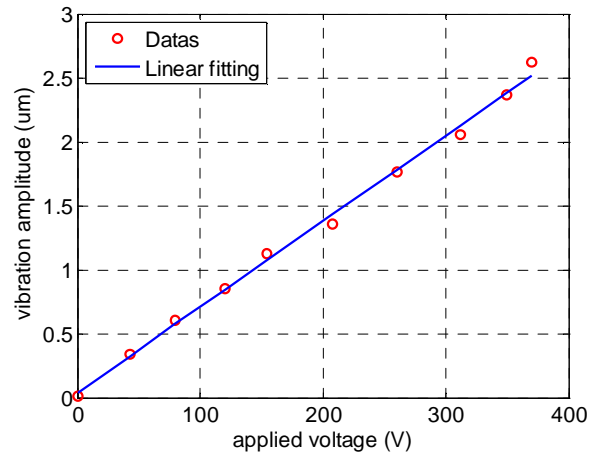

Fig. 7. The linear relation between the supply voltage and the vibration amplitude at the resonance.

We also recorded the response of the system at each step of variation of $V$. Despite the different voltage levels, it may be noticed that a first order behaviour was obtained as predicted by the modelling. From these characteristics we used ARX [24] for the identification method of $K_{1}$ and $\tau_{1}$ because it gives good results at low order. This identification method uses an estimation of the model parameters using the least-squares method to reduce error between data and model, the order of the model must be specified before the identification process. The results for each trial are presented in table 1 .

$$
\text { TABLE I }
$$

PARAMETERS OF THE IDENTIFIED TRANSFER FUNCTIONS

\begin{tabular}{llllll}
\hline \hline & & & & & Mean \\
\hline Voltage amplitude & 120 & 208 & 312 & 370 & \\
$\mathrm{~K}_{1}(\mathrm{pm} / \mathrm{V})$ & 7108 & 6547 & 6620 & 7103 & 6980 \\
$\tau_{1}(\mathrm{~ms})$ & 1.85 & 1.71 & 1.94 & 2.07 & 1.88 \\
\hline \hline
\end{tabular}

The variations of $\tau_{1}$ as a function of the voltage applied are plotted in Fig. 8.

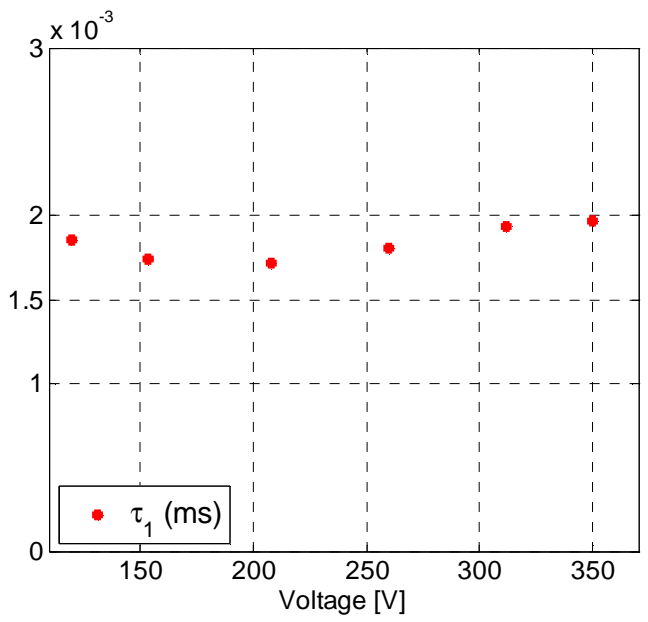

Fig. 8. Evolution of $\tau_{1}$ as a function of the applied voltage

The deviations of $\tau_{1}( \pm 6 \%)$ and $K_{1}( \pm 5 \%)$ are found to be sufficiently small to consider that they are constant for all voltage condition. 
2) Identification of the transfer function between $W_{q} / W_{d}$ and $\Delta \omega$

The identification of this second transfer function needs some experimental conditions, already explained in the modelling. In particular, for using linearization, we must ensure that:

- The system is excited in the vicinity of the resonance which makes $\Delta \omega / \omega_{0} \approx 0$.

- The real part of the vibration amplitude $W_{d}$ is controlled and constant, by using a control as detailed in the next section.

The transfer function (19) between $W_{q} / W_{d}$ and $\Delta \omega$ is identified by applying steps of $\Delta \omega$ as input, and measuring $W_{q} / W_{d}$ as output.

Fig. 9 represents the evolution of the quantity $W_{q}(p) / W_{d}$ as a function of $\Delta \omega$. Every $20 \mathrm{~ms}$, a decreasing step of $-77 \mathrm{rad} / \mathrm{s}$ of angular frequency is applied. At the same time, $W_{d}$ is maintained constant. Fig. 10 illustrates the relative error of the controlled $W_{d}$ in response to decreasing steps of the angular frequency to verify that it is maintained constant.
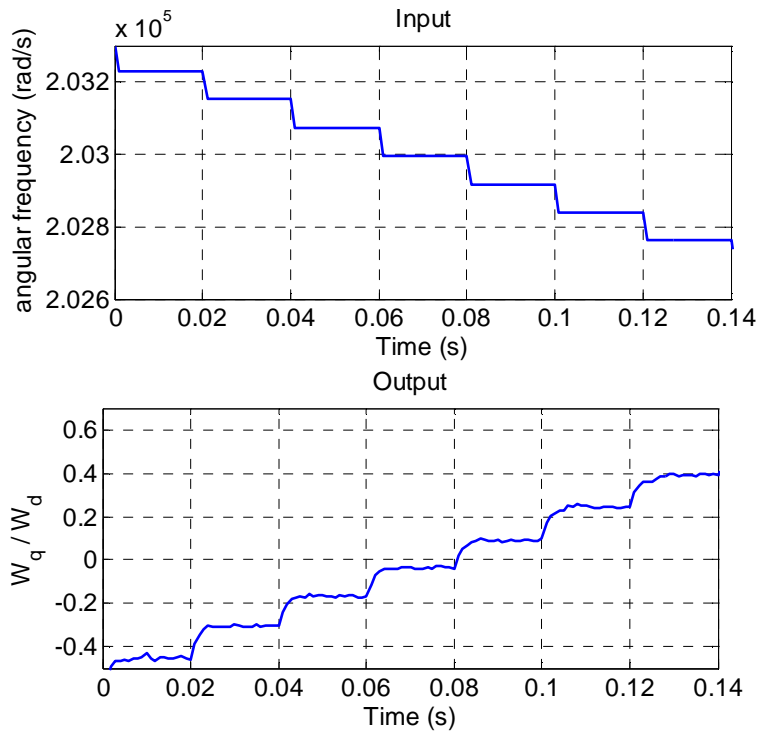

Fig. 9. Response of $W_{q} / W_{d}$ as a function of decreasing steps of the angular frequency.

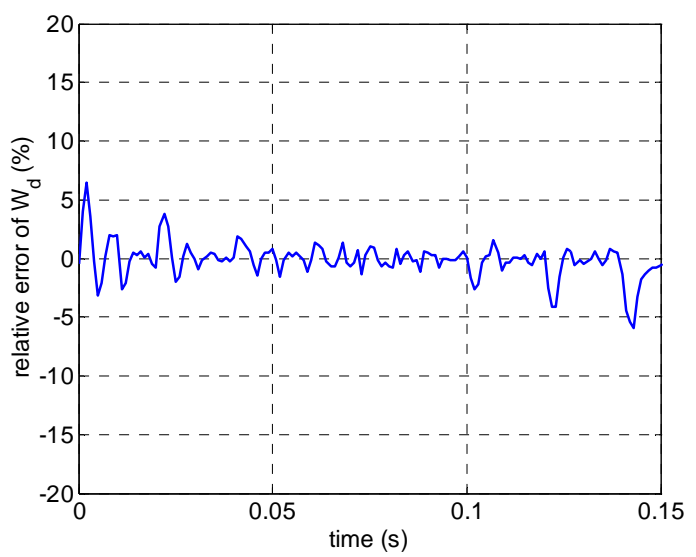

Fig. 10. Relative error of the controlled $W_{d}$ in response to steps of angular frequency $\left(W_{d \text { ref }}\right.$ is $2 \mu \mathrm{m}$ peak-peak).

In response to decreasing steps of the angular frequency $\Delta \omega$ from $\omega_{1}$ to $\omega_{2}$ around resonance, the quantity $W_{q}(p) / W_{d}$ increases following a first order behaviour. The identification of the transfer function presented in (19) gives the parameters $K_{2}$ and $\tau_{2}$ for each step. The two extremes and the medium experimental identification results are presented in the table 2 :

TABLE 2

PARAMETERS OF THE IDENTIFIED TRANSFER FUNCTIONS

\begin{tabular}{ccccc}
\hline \hline & & & & Mean \\
\hline$\omega_{1}(\mathrm{rad} / \mathrm{s})$ & 203229 & 202995 & 202839 & \\
$\omega_{2}(\mathrm{rad} / \mathrm{s})$ & 203151 & 202917 & 202761 & \\
$\mathrm{~K}_{2}\left(10^{-3}\right)$ & 1.9727 & 1.6876 & 1.8564 & $\mathrm{~K}_{2}=1.812$ \\
$\tau_{2}(\mathrm{~ms})$ & 1.5360 & 1.6504 & 1.8608 & $\tau_{2}=1.624$ \\
\hline \hline
\end{tabular}

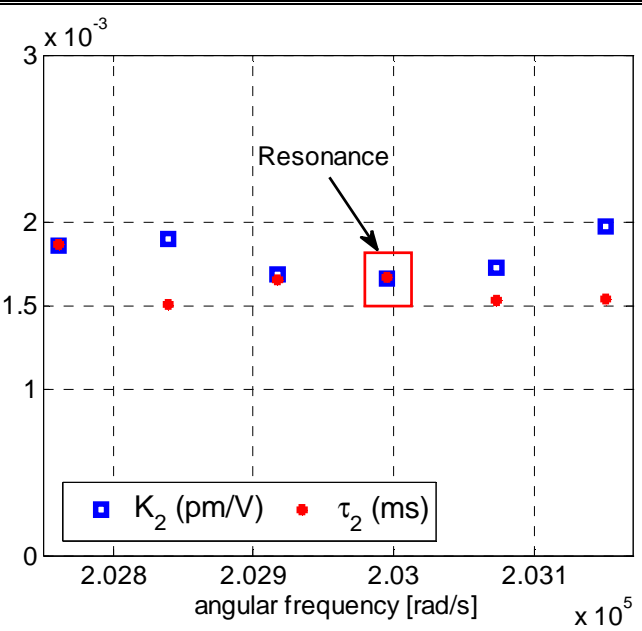

Fig. 11. Evolution of $\tau_{2}, K_{2}$ as a function of the angular frequency

Moreover, we have plotted some values of, $K_{2}$ and $\tau_{2}$ as a function of the angular frequency in the vicinity of the resonant frequency (Fig. 11). It can be stated that at the exact resonant angular frequency $(\omega=202995 \mathrm{rad} / \mathrm{s})$, this ratio between $K_{2}$ and $\tau_{2}$ is equal to 1 as expected by the equation (19).

Finally, the parameters of the function between $W_{q}(p) / W_{d}$ and $\Delta \omega$ are $K_{2}=1.81210^{-3}$ and $\tau_{2}=1.624 \mathrm{~ms}$.

The most important feature of the identification is that we validate by real measurement the validity of the model and approximations. We find that the two transfer functions have a similar constant time (respectively $\tau_{1}=1.88 \mathrm{~ms}$ and $\tau_{2}=1.62$ $\mathrm{ms})$. Moreover, the transfer function giving $W_{q}(p) / W_{d}$ as a function of $\Delta \omega$ has the static gain equal to its time constant. In the next section, a real time implementation of the two controllers will be achieved and thanks to an experimental measurement, a comparison between the closed loop control against the open loop one will be presented.

\section{CONTROL IMPLEMENTATION}

\section{A. Calculation of the controller coefficients}

The parameters of the controller are calculated through the identified transfer function. The two transfer functions are of 
first order type. A classical proportional integral controller is sufficient due to its low transfer function order; it can reduce the response time without any static error. In the following part, the method the parameters calculation is presented.

The structure of the controller is defined in (20) which contains two variable parameters $K_{p}$ and $K_{i}$ :

$$
P I_{\text {controller }}=K_{p}+\frac{K_{i}}{p}
$$

The classical closed loop control for a first order system $H(p)$ is represented in Fig. 12:

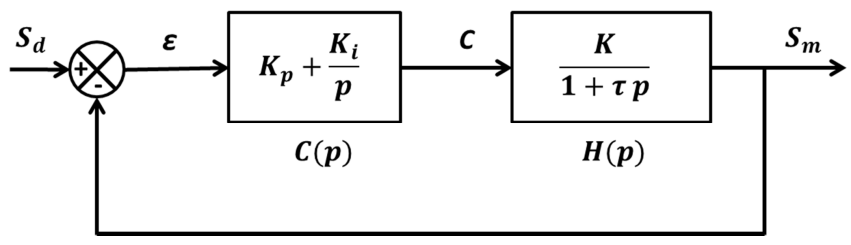

Fig. 12. Classical closed loop control for a first order transfer function using PI controller.

With $S_{d}$ is the desired output, $S_{m}$ is the measured output, $\varepsilon$ is the error between $S_{d}$ and $S_{m}$ and $C$ is the output of the controller. The characteristic equation of the closed-loop system shown in Fig. 7 is given by:

$$
\frac{S_{m}}{S_{d}}=\frac{C(p) H(p)}{1+C(p) H(p)}
$$

with

$$
C(p) H(p)=\left(\frac{K K_{i}}{1+\tau p}\right)\left(\frac{1+\frac{K_{p}}{K_{i}} p}{p}\right)
$$

We assume that $k_{p} / k_{i}=\tau$ to compensate the pole of the transfer function.

By equating the characteristic equation in closed loop with this assumption, we find a first order system between $S_{m}$ and $S_{d}$ given by:

$$
\frac{S_{m}}{S_{d}}=\frac{1}{1+\tau_{f} p}
$$

With $\tau_{f}=1 / K K_{i}$ is the desired time constant in closed-loop. The parameters of the controller can be calculated from $\tau, \tau_{f}$, and the parameters of the transfer function in open loop ( $K$ and $\tau$ ): for instance, the desired response time $t_{r 5 \%}$ can be imposed. For a first order function, it is equal to $3 \tau_{f}$ which leads to the equations of the controller parameters:

$$
\left\{\begin{array}{c}
K_{p}=\frac{3 \tau}{K t_{r 5 \%}} \\
K_{i}=\frac{K_{p}}{\tau}
\end{array}\right.
$$

This method will be used to control the identified transfer functions modeled in the section VI.

\section{B. Results for the two control loops}

The two controllers were implemented in the DSP with a sampling frequency of $1 \mathrm{kHz}$. The response time of the two controllers was chosen in order to accelerate the response time of the vibration amplitude to achieve about $2 \mathrm{~ms}$, otherwise it was $5 \mathrm{~ms}$ in open loop. The first series of experiments shows the response in open and closed loops of the vibration amplitude at the resonance and without any finger pressure (Fig. 13).
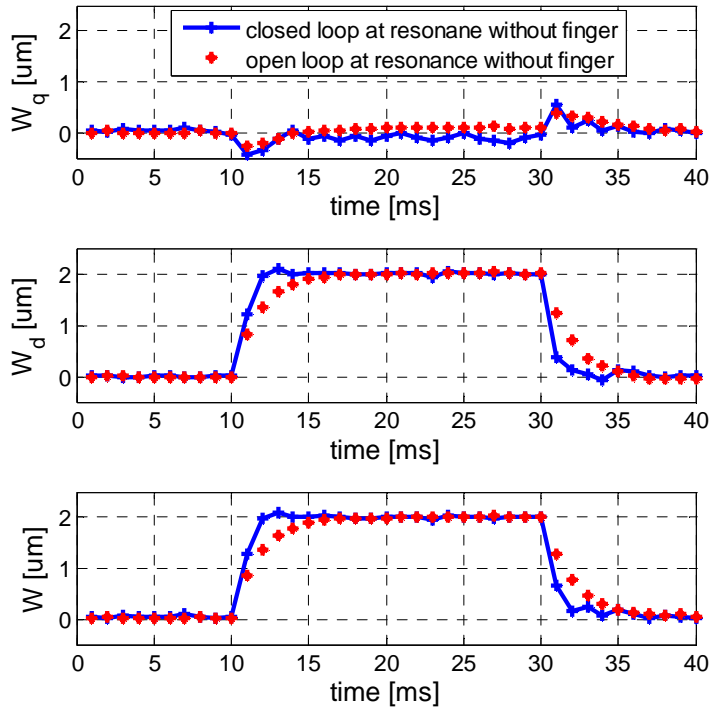

Fig. 13. Response of the system in open and closed loop control when exciting the system at resonance and without any finger pressure. Figures (a),(b) and (c) represent respectively the evolution of $W_{q}, W_{d}$ and $W$ which is the module of $W_{d}$ and $W_{q}$

The reference value for $W_{q}$ is zero, whereas the reference value for $W_{d}$ is $2 \mu \mathrm{m}$. By observing the response of $W_{d}$, we can conclude that the use of the closed loop configuration has an influence on the response time by decreasing it from 5 to 2 ms which may make the vibration more sensitive for the fingertip. The response of $W_{q}$ is almost zero both in close or open loop which is explained by the absence of external disturbance factors in this case. By repeating this experiment 30 times, the repeatability standard deviation was about $1.3 \%$ of the desired vibration amplitude of $2 \mu \mathrm{m}$.

The second series of experiments aims at evaluating the robustness of the controllers against temperature variation. The idea is to supply voltage at an initial frequency which corresponds to the resonant frequency at $33^{\circ} \mathrm{C}$ which was measured before doing the experiment $\left(\omega_{2}=202450 \mathrm{rad} / \mathrm{s}\right)$ whereas the real temperature during this experiment is about $24^{\circ} \mathrm{C}$ with the same excitation voltage comparing to the first experience. The validation of the control strategy was tested with an extreme possible condition of variation of temperature from $33^{\circ}$ to $24^{\circ}$ in order to validate the robustness of the controller. If the controller will work well with a minimum response time at this extreme condition, we will conclude that the system control strategy will work better when the temperature change is small and the control strategy will be 
validated. We observe in open loop control that $W_{q}$ becomes nonzero and $W_{d}$ decreases from the desired vibration amplitude $W=2 \mu \mathrm{m}$ to $1.6 \mu \mathrm{m}$. On the other hand, the closed loop control strategy ensures robustness against temperature variation with a low response time $\approx 2 \mathrm{~ms}$ and no more static error (Fig. 14). If we wanted to reach the same vibrating amplitude in open loop with this error on the temperature, we should provide a higher voltage supply.
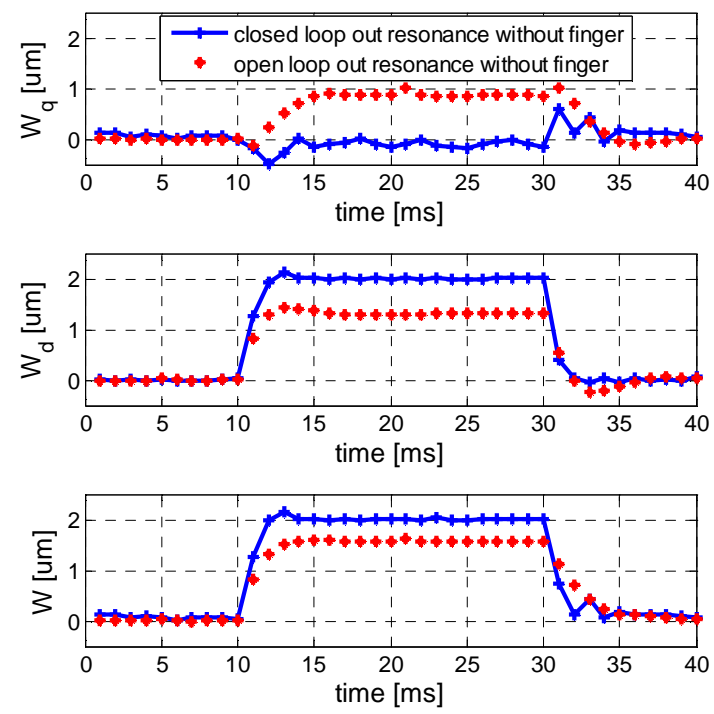

Fig. 14. Response of the system in open and closed loop control when exciting the system as if it was at $33^{\circ} \mathrm{C}$ whereas the actual temperature is $24^{\circ} \mathrm{C}$ and without any finger pressure. Figures (a), (b) and (c) represent respectively the evolution of $W_{q}, W_{d}$ and $W$ which is the module of $W_{d}$ and $W_{q}$ (see Fig. 5).

In the closed loop control, $W_{q}$ is nearly zero which proves that the excitation frequency of the system has converged towards the resonant frequency (Fig. 15).

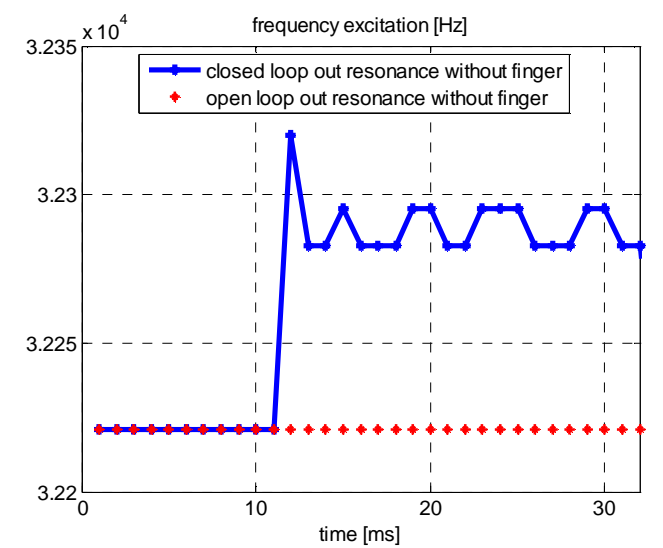

Fig. 15. Frequency excitation behaviour in open loop and closed loop control when the system is excited out of resonance. At the instant $11 \mathrm{~ms}$, the frequency controller in switched on and converges to the resonant frequency of the temperature $24^{\circ} \mathrm{C}$ which is $32300 \mathrm{kHz}$ (Fig. 4). In red (the dotted line), for open loop condition, the excitation frequency is still fixed to the resonant frequency of $33^{\circ} \mathrm{C}$.

In order to assess the system performance against the two disturbing factors at the same time (finger pressure and temperature variation), a third experience has been performed.
The experimental conditions are similar to the previous experiment one (excite the system at the $33^{\circ} \mathrm{C}$ resonant frequency), but this time, we add a finger sliding on the active surface of the plate with a pressure about $2 \mathrm{~N}$, the finger has to move back and forth on the tactile plate to feel stimulation, the finger pressure is shown in a screen in front of the experimenter to help him maintaining it constant. The operation of these two conditions of temperature validation and touch force is done to evaluate the robustness of the control law in extreme conditions to validate it. It is shown that the open loop control is sensitive to the external disturbing factors. The closed loop control maintains the vibration amplitude constant in a short response time.
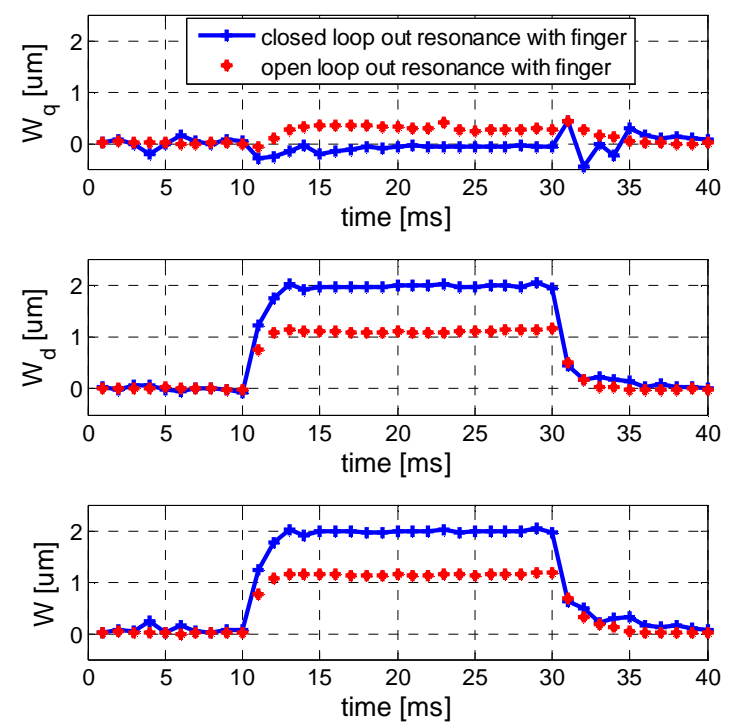

Fig. 16. Response of the system in open and closed loop control when exciting the system as if it was at $33^{\circ} \mathrm{C}$ whereas the actual temperature is $24^{\circ} \mathrm{C}$ and with approximately $2 \mathrm{~N}$ finger pressure. Figures (a),(b) and (c) represent respectively the evolution of $W_{q}, W_{d}$ and $W$ which is the module of $W_{d}$ and $W_{q}$ (see Fig. 5).

These experiments confirmed the importance of the closed loop control of the vibration amplitude to ensure a stable level of squeeze film. The psychophysics test may be achieved to compare the open loop, the first control strategy [10] and the results of this work together. In our current work [25], the focus is put on the control of the friction by the vibration amplitude in order to evaluate the relation between friction and level of squeeze film effect and to compensate the difference between users.

\section{CONCLUSION}

In this paper, we proposed a new approach to control the wave amplitude of the vibration created for tactile stimulation. The objective was to make the effect of the tactile feedback insensitive to external disturbances such as the fingertip pressure and the temperature variation. For that aim, a new modelling has been developed analytically in a rotating reference frame. By aligning the rotating frame on the voltage, we obtain two first order type equations, for the control of the 
vibration amplitude and for the tracking of the resonant frequency which has been also validated experimentally in the useful range of work. The identification has been performed and two simultaneous closed loop controls of the vibration amplitude and of the frequency have been implemented. The closed loop results show a high robustness of the new control strategy against the two disturbing factors.

\section{REFERENCES}

[1] O. Bau, I. Poupyrev, A. Israr, and C. Harrison, "TeslaTouch: electrovibration for touch surfaces," in Proceedings of the 23nd annual ACM symposium on User interface software and technology, New York, NY, USA, 2010, pp. 283-292.

[2] K. A. Kaczmarek, K. Nammi, A. K. Agarwal, M. E. Tyler, S. J. Haase, and D. J. Beebe, "Polarity effect in electrovibration for tactile display," IEEE Trans. Biomed. Eng., vol. 53, no. 10, pp. 2047-2054, Oct. 2006.

[3] T. Watanabe and S. Fukui, "A method for controlling tactile sensation of surface roughness using ultrasonic vibration," in IEEE International Conference on Robotics and Automation, 1995, vol. 1, pp. 1134-1139.

[4] C. Winter, Y. Civet, and Y. Perriard, "Optimal design of a squeeze film actuator for friction feedback," in Electric Machines Drives Conference (IEMDC), 2013 IEEE International, 2013, pp. 14661470 .

[5] M. Biet, F. Giraud, and B. Lemaire-Semail, "Implementation of tactile feedback by modifying the perceived friction," Eur. Phys. J. - Appl. Phys., vol. 43, no. 01, pp. 123-135, 2008.

[6] R. J. E. Merry, J. L. Holierhoek, M. J. G. van de Molengraft, and M Steinbuch, "Gain Scheduling Control of a Walking Piezo Actuator," IEEEASME Trans. Mechatron., vol. 19, no. 3, pp. 954-962, Jun. 2014.

[7] F. Giraud, M. Amberg, and B. Lemaire-Semail, "Design and control of a haptic knob," Sens. Actuators Phys., vol. 196, pp. 78-85, 2013.

[8] Z. Yao, Z. N. Guo, Y. J. Zhang, Y. Deng, and W. T. Zhang, "Research on the Frequency Tracking in Rotary Ultrasonic Machining," Procedia CIRP, vol. 6, pp. 556-560, 2013.

[9] Y. Kuang, Y. Jin, S. Cochran, and Z. Huang, "Resonance tracking and vibration stablilization for high power ultrasonic transducers," Ultrasonics, vol. 54, no. 1, pp. 187-194, Jan. 2014.

[10] W. Ben Messaoud, B. Lemaire-Semail, M.-A. Bueno, M. Amberg, and F. Giraud, "Closed-Loop Control for Squeeze Film Effect in Tactile Stimulator," presented at the Proceedings of the 2014 international Conference and exhibition on new actuators and drives, Actuator 2014, Bremen, Germany, 2014, pp. 245-248.

[11] C. A. Desoer, "The bang bang servo problem treated by variational techniques," Inf. Control, vol. 2, no. 4, pp. 333-348, Dec. 1959.

[12] F. Giraud, B. Semail, and J.-T. Audren, "Analysis and phase control of a piezoelectric traveling-wave ultrasonic motor for haptic stick application," IEEE Trans. Ind. Appl., vol. 40, no. 6, pp. 1541-1549, Nov. 2004.

[13] F. Pigache, F. Giraud, and B. Lemaire-Semail, "Modelling and identification of a planar standing wave ultrasonic motor," Eur. Phys. J. - Appl. Phys., vol. 34, no. 01, pp. 55-65, 2006.

[14] F. Giraud, C. Giraud-Audine, M. Amberg, and B. Lemaire-Semail, "Vector control method applied to a traveling wave in a finite beam," IEEE Trans. Ultrason. Ferroelectr. Freq. Control, vol. 61, no. 1, pp. 147-158, Jan. 2014.

[15] W. Leonhard, Control of Electrical Drives. Springer, 1985.

[16] P. Cossutta, M. P. Aguirre, M. A. Engelhardt, A. Cao, and M. I. Valla "High speed fixed point DSOGI PLL implementation on FPGA for synchronization of grid connected power converters," in 2014 IEEE 23rd International Symposium on Industrial Electronics (ISIE), 2014, pp. 1372-1377.

[17] S. B. Backus and A. M. Dollar, "Robust Resonant Frequency-Based Contact Detection With Applications in Robotic Reaching and Grasping," IEEEASME Trans. Mechatron., vol. 19, no. 5, pp. 15521561, Oct. 2014.
[18] I. Darian-Smith, "The Sense of Touch: Performance and Peripheral Neural Processes," in Comprehensive Physiology, John Wiley \& Sons, Inc., 2011.

[19] F. Giraud, M. Amberg, B. Lemaire-Semail, and G. Casiez, "Design of a transparent tactile stimulator," in 2012 IEEE Haptics Symposium (HAPTICS), 2012, pp. 485-489.

[20] F. Giraud, M. Amberg, and B. Lemaire-Semail, "Merging two tactile stimulation principles: electrovibration and squeeze film effect," in World Haptics Conference (WHC), 2013, 2013, pp. 199-203.

[21] M. Amberg, F. Giraud, B. Semail, P. Olivo, G. Casiez, and N. Roussel, "STIMTAC: a tactile input device with programmable friction," in Proceedings of the 24th annual ACM symposium adjunct on User interface software and technology, New York, NY, USA, 2011, pp. 7-8.

[22] M. Wiertlewski, D. Leonardis, D. J. Meyer, M. A. Peshkin, and J. E. Colgate, "A High-Fidelity Surface-Haptic Device for Texture Rendering on Bare Finger," in Haptics: Neuroscience, Devices, Modeling, and Applications, M. Auvray and C. Duriez, Eds. Springer Berlin Heidelberg, 2014, pp. 241-248.

[23] C.-M. Lin, "Temperature Compensation of Aluminum Nitride Lamb Wave Resonators Utilizing the Lowest-Order Symmetric Mode," EECS Department, University of California, Berkeley, 2012.

[24] L. Ljung, "System Identification," in Signal Analysis and Prediction, A. Procházka, J. Uhlír, P. W. J. Rayner, and N. G. Kingsbury, Eds. Birkhäuser Boston, 1998, pp. 163-173.

[25] W. Ben Messaoud, M. Amberg, B. Lemaire-Semail, F. Giraud, and M.-A. Bueno, "High fidelity closed loop controlled friction in SMARTTAC tactile stimulator," Accepted at the 17th European Conference on Power Electronics and Applications (EPE'15-ECCE Europe), Geneva, 2015.

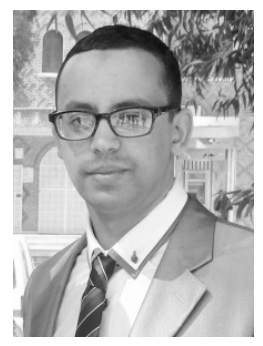

Wael Ben Messaoud received his M.Eng. degree in electrical engineering in 2011 and his M.S degree in Automatic control and Industrial Computing in 2012 from the National School of Engineers of Sfax ENIS (Tunisia). He is currently $\mathrm{PhD}$ student in electrical engineering from November 2012. His thesis is in collaboration between L2EP Lab. University Lille 1 and LPMT Lab. University of Haute Alsace, France. His work is focused on the modelling and control of haptic feedback devices.

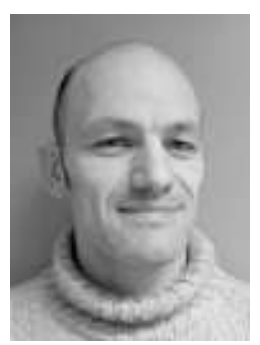

Frédéric Giraud received the B.S. degree in 1995 from Paris-XI University, graduated from the Ecole Normale Supérieure de Cachan, France in 1996 in electrical engineering, and received the M.S. degree in 1997 from the Institut National Polytechnique de Toulouse and the Ph.D. degree in 2002 from the University Lille $1 . \mathrm{He}$ is a member of the electrical engineering and power electronics laboratory of Lille, where he works as an Associate Professor. His research deals with the modelling and control of piezoelectric actuators.

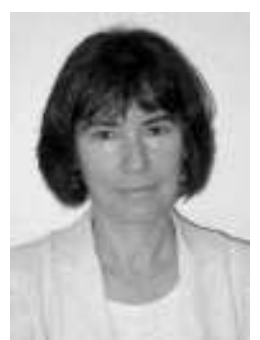

Betty Lemaire-Semail received the Ph.D. degree in 1990 from the University of Paris XI, Orsay. From 1990 to 1998 , she was an assistant professor at the Ecole Centrale of Lille and she is now a professor at the University Lille 1 . She is a member of the electrical engineering and power 
electronics laboratory of Lille and head of the research axis on the control of electrical systems. She has studied electromagnetic motors, and her main field of interest now deals with the modelling and control of piezoelectric actuators for positioning and force feedback applications.

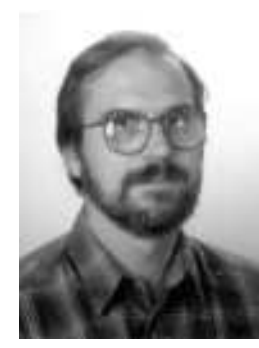

Michel Amberg has been teaching electronics at the University of Lille, France. He graduated from the Ecole Normale Supérieure de Cachan, France in 1981. He has tutored more than a hundred bachelor's students during their projects in the field of telecommunications, computer science, and electronics. He is now a research engineer at IRCICA, and works on the electronic design of tactile devices.

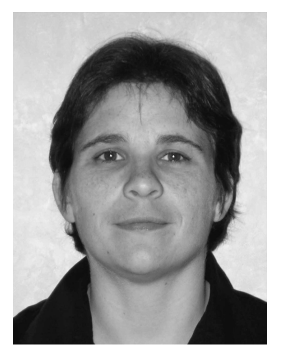

Marie-Ange Bueno received her $\mathrm{PhD}$ degree from the University of Haute Alsace France_in 1995. Since 1997, she has been an assistant professor in fiber science and mechanics, and since 2005 a full professor at the Ecole Nationale Supérieure d'Ingénieurs Sud Alsace. She has worked since 1992 on tribology of fibrous materials with a special interest to tactile feeling. She is member of the French Mechanical Society (AFM) and the Fiber Society. 